\title{
Thoracoscopic segmentectomy compares favorably with thoracoscopic lobectomy for patients with small stage I lung cancer
}

\author{
Mark Shapiro, MD, ${ }^{\mathrm{a}}$ Todd S. Weiser, MD, ${ }^{\mathrm{b}}$ Juan P. Wisnivesky, MD, MPH, ${ }^{\mathrm{c}}$ Cynthia Chin, MD, ${ }^{\mathrm{b}}$ Michael Arustamyan, BA, \\ and Scott J. Swanson, MD ${ }^{\mathrm{d}}$
}

\begin{abstract}
Objective: As thoracoscopic lobectomy becomes widely applied for treatment of non-small cell lung cancer, thoracoscopic segmentectomy remains controversial for patients with small stage I lung cancers. Questions remain regarding safety, morbidity, mortality, and recurrence rate. This study compared outcomes between thoracoscopic segmentectomy and lobectomy.
\end{abstract}

\begin{abstract}
Methods: Retrospective review was undertaken of patients who underwent thoracoscopic segmentectomy or lobectomy for clinical stage I non-small cell lung cancer between January 2002 and February 2008. Indications for segmentectomy were tumor smaller than $3 \mathrm{~cm}$, limited pulmonary reserve, comorbidities, and peripheral tumor location.
\end{abstract}

Results: Thirty-one patients underwent segmentectomy and 113 underwent lobectomy. Patients after segmentectomy had worse mean forced expiratory volume in 1 second than after lobectomy $(83 \%$ vs $92 \%, P=.04)$. There were no differences in mean number of nodes (10) and nodal stations (5) resected. Segmentectomy and lobectomy groups had similar median chest tube durations ( 2 vs 3 days, $P=.18$ ), stays (both 4 days), total complications, recurrence rates, and survivals at mean follow-ups of 22 and 21 months, respectively. Lobectomy group had $130-$ day death; segmentectomy group had none. There were $5(17.2 \%)$ recurrences after segmentectomy and 23 $(20.4 \%)$ after lobectomy $(P=.71)$, with locoregional recurrence rates of $3.5 \%$ and $3.6 \%$, respectively.

Conclusion: Thoracoscopic segmentectomy is a safe option for experienced thoracoscopic surgeons treating patients with small stage I lung cancers. No significant difference in oncologic outcome was seen between thoracoscopic segmentectomy and thoracoscopic lobectomy. Lymph node dissection could be performed as effectively during segmentectomy as lobectomy.

Lobectomy is the standard surgical treatment for early stage lung cancer. In 1973, Jensik and colleagues ${ }^{1}$ published a study suggesting that segmental resection was equivalent to lobectomy and represented an adequate operation for small stage I non-small cell lung cancers (NSCLCs). This publication started a debate regarding the optimal surgical approach for early stage NSCLC. Results of a randomized trial published in 1995 showed that limited pulmonary resection for stage IA NSCLC did not result in improved morbidity, mortality, or postoperative pulmonary function and was associated with higher rates of locoregional recurrence and death relative to lobectomy. ${ }^{2}$ Lobectomy, however, may not be the best option for patients with poor cardiopulmonary function. Recently, as a result of an increasing incidence of small lung tumors, there has been renewed interest in the use of anatomic segmentectomy, especially for patients unable to tolerate lobectomy because of compro-

\footnotetext{
From the Department of Surgery, ${ }^{\mathrm{a}}$ the Division of Thoracic Surgery, ${ }^{\mathrm{b}}$ and the Department of Medicine, ${ }^{\mathrm{c}}$ The Mount Sinai Medical Center, New York, NY, and the Division of Thoracic Surgery, ${ }^{\mathrm{d}}$ Brigham and Women's Hospital, Boston, Mass.

Received for publication Oct 25, 2008; revisions received Dec 18, 2008; accepted for publication Feb 1, 2009.

Address for reprints: Scott J. Swanson, MD, Division of Thoracic Surgery, Brigham and Women's Hospital, Peter Bent Brigham Building 239, 75 Francis St, Boston, MA 02115 (E-mail: sjswanson@partners.org).

J Thorac Cardiovasc Surg 2009;137:1388-93

$0022-5223 / \$ 36.00$

Copyright (c) 2009 by The American Association for Thoracic Surgery doi:10.1016/j.jtcvs.2009.02.009
}

mised medical condition. Several recently published studies have shown that segmentectomy can be performed safely without compromising oncologic results. ${ }^{3-7}$

Advantages of a minimally invasive approach to lobectomy-video-assisted thoracic surgery (VATS) lobectomy-have been previously documented in the literature. These include decreased postoperative pain, shorter recovery, preserved pulmonary function, decreased inflammatory response, and improved tolerance of chemotherapy, with equivalent oncologic outcomes for VATS lobectomy as for open lobectomy. ${ }^{8-15}$ VATS lobectomy is becoming the standard of care.

Despite the growing acceptance of VATS lobectomy, VATS segmentectomy remains highly controversial as a choice for treatment of small lung tumors. In addition to concerns about increased locoregional recurrence, potential arguments against VATS segmentectomy include higher rates of complications and inadequate nodal dissection because of the high complexity of the procedure. ${ }^{5,16}$ Combining VATS and limited resection in the form of a VATS segmentectomy, however, may give the benefits of a VATS approach to patients who need a sublobar resection. To date, only a few studies have been published investigating the outcomes of VATS segmentectomy in patients with NSCLC, and these have generally used a thoracotomy approach for the segmentectomy as their comparison group. There is a lack of published studies examining the recurrence rate after VATS 


\section{Abbreviations and Acronyms \\ NSCLC $=$ non-small cell lung cancer \\ VATS $=$ video-assisted thoracic surgery}

segmentectomy relative to that after VATS lobectomy. This study examined the role of VATS segmentectomy for treatment of small, early stage NSCLC. We compared morbidity, recurrence, and survival between patients who underwent VATS segmentectomy and similarly staged patients who underwent VATS lobectomy.

\section{MATERIALS AND METHODS}

From the Lung Cancer Database at the Mount Sinai Medical Center, a retrospective review was conducted of all patients who underwent VATS anatomic segmentectomy for clinical stage I NSCLC from January 2002 to February 2008. Comparison was made with a series of patients who underwent VATS lobectomy for similarly sized NSCLC. Inpatient and outpatient charts were reviewed, and information was collected with a standardized questionnaire. Data included patients' demographic data, comorbidities, operative and hospital courses, pathologic staging, and postoperative followup. Patients were contacted for additional follow-up information, also with a standardized questionnaire. Operative mortality was defined as death during the hospitalization for the pulmonary resection or within 30 days of the procedure, whichever was longer. Pathologic cancer staging was done in accordance with the guidelines set forth by the American Joint Committee on Cancer.

Before surgical resection, all patients underwent preoperative staging with computed tomographic scanning, with or without positron emission tomography, and pulmonary function testing. Additional diagnostic tests were performed according to patient symptoms and clinical findings. Mediastinoscopy was not used routinely in the preoperative evaluation of these patients and was performed only on patients with lymph node enlargement greater than $1 \mathrm{~cm}$ on computed tomographic scan or positive lymph node findings on positron emission tomographic scan. In cases where mediastinoscopy was not done, however, ipsilateral thoracoscopic mediastinal node evaluation (N2) was performed at the outset of the lobectomy or segmentectomy. For patients who were found intraoperatively by frozen-section analysis of the lymph nodes to have N2 disease, the operation was terminated and induction therapy was carried out. These patients were not included in this study. Indications for segmentectomy rather than lobectomy were small tumor size $(<3 \mathrm{~cm})$, limited pulmonary reserve with extensive comorbidities, and peripheral location of the tumor.

Bronchoscopy was performed on all patients after intubation and before repositioning to ensure that the segmental bronchus was free of disease. For our thoracoscopic approach, three incisions were used. Full description of this technique has been previously published. ${ }^{10}$ The avoidance of rib spreading was critical to the definition of a VATS approach. If rib spreading was carried out, then the operation was considered a conversion to thoracotomy. For a resection to be considered a segmentectomy, individual ligation of the segmental bronchus and vascular structures was essential. Completion of the fissure was done with endostaplers after visualization of the segmental boundaries, and all patients underwent complete hilar and mediastinal node dissection.

\section{Statistical Analysis}

The Student $t$ test and the Wilcoxon test were used for continuous data, and the $\chi^{2}$ test was used for dichotomous data. Disease-free and overall survivals were estimated with the Kaplan-Meier method. Disease-free survival was defined as the time from surgery to the first diagnosis of local, regional, or distant disease recurrence or until the last follow-up. Overall survival was defined as the time from surgery to death from any cause or last follow-up. To avoid calculating survival from a small number of observations, data for survival curves were censored at 48 months for surviving patients. We used Cox regression analysis to identify predictors of recurrence after resection of stage I disease. We tested the assumption of proportionality of hazards with the log-log plotting of survival among appropriate groups. Power calculation showed that given the 28 events observed in the population, the study had $80 \%$ power to detect whether segmentectomy is associated with at least a 3.5-times increased hazard of recurrence. The SAS 9.0 statistical package (SAS Institute, Inc, Cary, NC) was used for all statistical analyses. The institutional review board of the Mount Sinai School of Medicine approved this study.

\section{RESULTS}

During the stated period, 31 patients underwent VATS segmentectomy and 113 underwent VATS lobectomy for similarly sized and staged early stage NSCLC. Patient and tumor characteristics are summarized in Table 1. The two groups were similar in age and stage of lung cancer; however, the results of pulmonary function tests were significantly worse in the VATS segmentectomy group than in the VATS lobectomy group (forced expiratory volume in 1 second $83 \%$ vs $92 \%, P=.04$ ). Also, $61 \%$ of patients in the VATS segmentectomy group had pulmonary comorbidities before surgery, and $48 \%$ had evidence of significant cardiovascular disease. At the time of surgery, the average exposure to smoking in the VATS segmentectomy group was 47.8 pack-years, versus 34.4 pack-years in the VATS lobectomy group. Three patients in the VATS segmentectomy group had undergone pulmonary resections on the opposite lung within 2 years before the index surgery ( 2 for synchronous lesions and 1 for metachronous lesions).

There were 2 patients in VATS segmentectomy group with stage IIIA disease on the basis of positive mediastinal nodes found on final pathologic evaluation (level 5 in 1 patient and level 7 in the other), despite negative results of frozen-section evaluation. Moreover, 1 patient had disease staged as IIIB because final pathologic examination identified an unsuspected satellite lesion in the specimen. Mean tumor size in the VATS segmentectomy group was significantly smaller than in the VATS lobectomy group (2.1 vs $2.6 \mathrm{~cm}, P=.02$; Table 1 ).

The distribution of the segmentectomies performed is shown in Table 2. Seven patients in VATS segmentectomy group had mediastinoscopy done, either as a separate procedure or at the same time of the pulmonary resection. There was no difference in the number of nodes or the number of nodal stations resected. Both groups of patients on average underwent dissection of 5 nodal stations, resulting in an average of 10 lymph nodes available for pathologic evaluation and staging. Six patients in the VATS segmentectomy group underwent additional wedge resections for benign lesions as part of the procedure, and 1 patient underwent extended segmentectomy because of proximity of the tumor to the edge of the segment. There were 4 conversions to minithoracotomy 
TABLE 1. Demographic and clinical patient characteristics

\begin{tabular}{lccc}
\hline \multicolumn{1}{c}{ Variable } & $\begin{array}{c}\text { Segmentectomy } \\
(\mathbf{n}=\mathbf{3 1})\end{array}$ & $\begin{array}{c}\text { Lobectomy } \\
(\mathbf{n = 1 1 3})\end{array}$ & $\begin{array}{c}\boldsymbol{P} \\
\text { value }\end{array}$ \\
\hline Age, years (mean $\pm \mathrm{SD})$ & $65 \pm 9.5$ & $69 \pm 10.6$ & .11 \\
Sex (male/female) & $8: 23$ & $54: 59$ & .03 \\
Forced expiratory volume in & $83 \pm 22.0$ & $92 \pm 20.1$ & .04 \\
$\quad$ 1 s (\%, mean $\pm \mathrm{SD})$ & & & \\
Tumor size (cm, mean $\pm \mathrm{SD})$ & $2.1 \pm 0.9$ & $2.6 \pm 1.2$ & .02 \\
Histologic type (No.) & & & .003 \\
Adenocarcinoma & $19(61.3 \%)$ & $88(77.9 \%)$ & \\
Squamous cell & $3(9.7 \%)$ & $17(15.0 \%)$ & \\
Other & $7(22.6 \%)$ & $8(7.1 \%)$ & \\
Hamartoma & $2(6.4 \%)$ & 0 & \\
Stage* (No.) & & & .37 \\
IA & $20(69.0 \%)$ & $66(58.4 \%)$ & \\
IB & $5(17.2 \%)$ & $34(30.1 \%)$ & \\
IIA & $1(3.5 \%)$ & $4(3.5 \%)$ & \\
IIB & 0 & $4(3.5 \%)$ & \\
III or IV & $3(10.3 \%)$ & $5(4.5 \%)$ & \\
\hline
\end{tabular}

*Among patients treated for clinical stage I non-small cell lung cancer, $\mathrm{n}=29$ for segmentectomy and $\mathrm{n}=113$ for lobectomy.

in the VATS segmentectomy group ( 1 for bleeding, 3 for anatomic considerations). In the patient with intraoperative bleeding, hemostasis was achieved with a sponge stick before conversion to a minithoracotomy. One unit of blood was given to this patient during the surgery; however, he did not have any intraoperative hemodynamic instability.

Postoperative recovery and follow-up results were similar between the groups. Chest tube duration, hospital stay, and number of complications did not differ between the groups (Table 3). Specific complications after each procedure are listed in Table 4 . There was 1 death within 30 days recorded in the VATS lobectomy group, with none in VATS segmentectomy group (Table 3). There were no differences in the overall and site-specific rates of recurrence. The locoregional recurrence rate was low in both groups, with 1 case $(3.5 \%)$ in the VATS segmentectomy group and $4(3.6 \%)$ in the VATS lobectomy group. Overall and disease-free survivals were also similar between the groups (Table 3, Figure 1). Multivariate analysis also showed no significant difference in rate of recurrence between two types of resection (Table 5). To adjust for difference in tumor size in our

TABLE 2. Type of segmental resection by anatomic location

\begin{tabular}{lrr}
\hline & No. & \multicolumn{1}{c}{$\%$} \\
\hline Lingulectomy & 2 & $6.5 \%$ \\
Left LL superior segmentectomy & 3 & $9.7 \%$ \\
Lingula-sparing upper lobectomy & 17 & $54.8 \%$ \\
Right LL superior segmentectomy & 7 & $22.6 \%$ \\
Left LL composite basilar segmentectomy & 1 & $3.2 \%$ \\
Right LL composite basilar segmentectomy & 1 & $3.2 \%$ \\
Total & 31 & $100 \%$ \\
\hline
\end{tabular}

$L L$, Lower lobe.
TABLE 3. Recovery and follow-up

\begin{tabular}{llcc}
\multicolumn{1}{c}{ Variable } & $\begin{array}{c}\text { Segmentectomy } \\
(\mathbf{n = 3 1})\end{array}$ & $\begin{array}{c}\text { Lobectomy } \\
(\mathbf{n}=\mathbf{1 1 3})\end{array}$ & $\begin{array}{c}\boldsymbol{P} \\
\text { value }\end{array}$ \\
\hline Mean follow-up (mo, mean $\pm \mathrm{SD})$ & $22 \pm 16.6)$ & $21 \pm 12.6)$ & .83 \\
Chest tube duration (d, median & $2(1-33)$ & $3(2-35)$ & .18 \\
$\quad$ and range) & & & \\
Stay (d, median and range) & $4(1-98)$ & $4(3-34)$ & .10 \\
Complications (No.) & $8(25.8 \%)$ & $30(26.6 \%)$ & .82 \\
$\quad$ Major & $3(9.7 \%)$ & $7(6.2 \%)$ & \\
$\quad$ Minor & $5(16.1 \%)$ & $23(20.4 \%)$ & \\
Perioperative death (30-d, No.) & 0 & 1 & \\
Recurrence* (No.) & $5(17.2 \%)$ & $23(20.4 \%)$ & .71 \\
$\quad$ Locoregional & $1(3.5 \%)$ & $4(3.6 \%)$ & \\
Distant & $3(10.3 \%)$ & $13(11.5 \%)$ & \\
Both & $1(3.5 \%)$ & $5(5.3 \%)$ & \\
\hline
\end{tabular}

*Among patients treated for clinical stage I non-small cell lung cancer, $\mathrm{n}=29$ for segmentectomy and $\mathrm{n}=113$ for lobectomy.

groups, the analysis was repeated for patients with only stage IA cancer (mean tumor size in VATS segmentectomy and VATS lobectomy groups $1.7 \mathrm{~cm}$ and $1.8 \mathrm{~cm}$, respectively); this analysis also did not show any significant difference between the two types of resection $(P=.850)$.

The impact of size of tumor in patients with stage I NSCLC on recurrence after VATS segmentectomy was analyzed as well. Figure 2 shows a trend toward increased recurrence among patients with tumors larger than $2 \mathrm{~cm}$, but this trend did not reach statistical significance $(P=.33)$.

\section{DISCUSSION}

Our study analyzed the postoperative outcomes of patients undergoing a VATS segmentectomy. It is the only study to date to compare morbidity, recurrence, and survival data between patients undergoing VATS segmentectomy and VATS lobectomy. We have demonstrated that VATS segmentectomy can be performed safely with excellent

TABLE 4. Postoperative complications

\begin{tabular}{|c|c|c|c|c|}
\hline \multirow[b]{2}{*}{ Complication } & \multicolumn{2}{|c|}{ Segmentectomy } & \multicolumn{2}{|c|}{ Lobectomy } \\
\hline & No. & $\%$ & No. & $\%$ \\
\hline Atrial fibrillation* & $0 \%$ & $0 \%$ & $12 \%$ & $10.6 \%$ \\
\hline Effusion $\dagger$ & 1 & $3.2 \%$ & $0 \%$ & $0 \%$ \\
\hline Empyemał & 0 & $0 \%$ & $2 \%$ & $1.8 \%$ \\
\hline Respiratory failure $\S$ & 2 & $6.5 \%$ & $1 \%$ & $0.9 \%$ \\
\hline Vocal cord paralysis & 0 & $0 \%$ & $1 \%$ & $0.9 \%$ \\
\hline Air leak $(>5 \mathrm{~d})$ & 4 & $12.9 \%$ & $11 \%$ & $9.7 \%$ \\
\hline Pneumonia & 1 & $3.2 \%$ & $1 \%$ & $0.9 \%$ \\
\hline Clostridium difficile & 0 & $0 \%$ & $1 \%$ & $0.9 \%$ \\
\hline Death & 0 & $0 \%$ & $1 \%$ & $0.9 \%$ \\
\hline Total & 8 & $25.8 \%$ & $30 \%$ & $26.6 \%$ \\
\hline
\end{tabular}



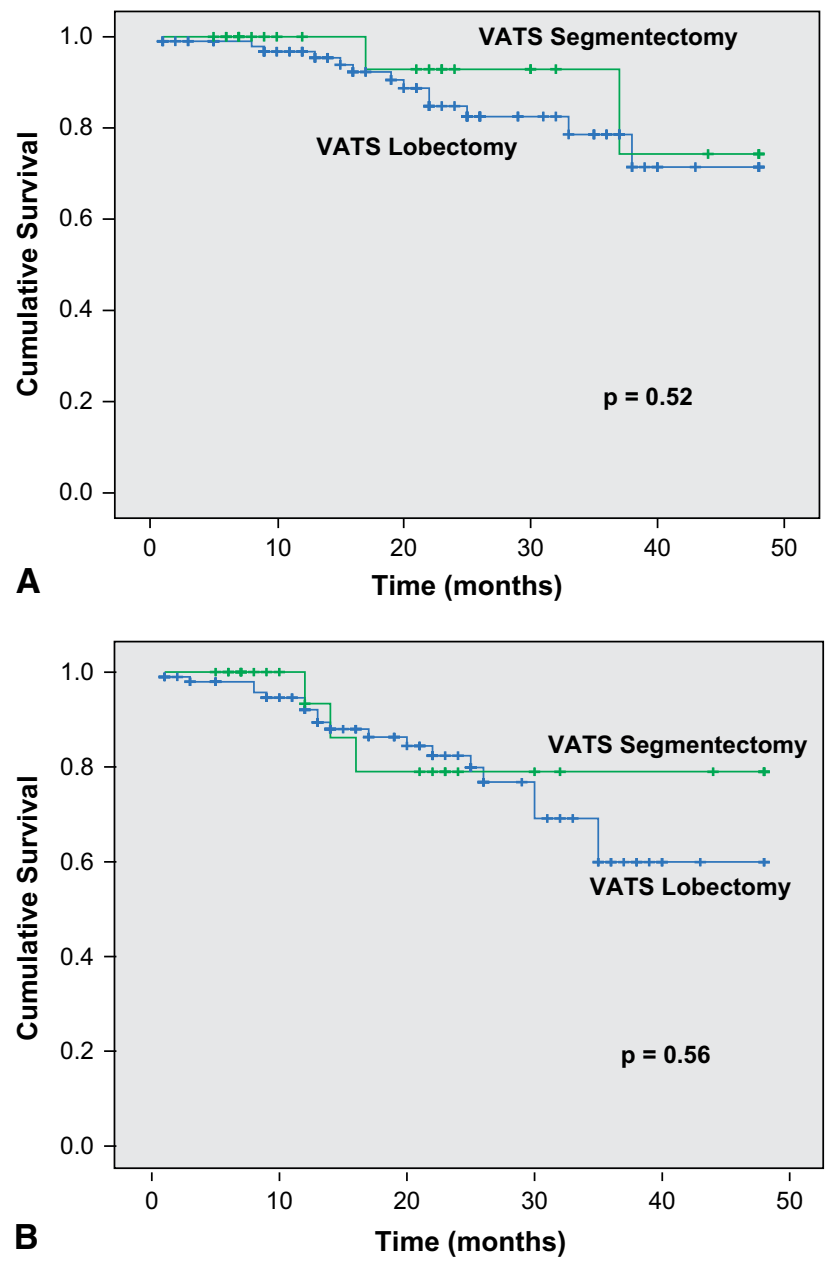

FIGURE 1. Survivals according to type of resection in patients with pathologic stage I non-small cell cancer, video-assisted thoracoscopic surgery segmentectomy (VATS Segmentectomy) versus video-assisted thoracoscopic surgery (VATS lobectomy). A, Overall survival; B, disease-free survival.

outcomes and a low rate of locoregional recurrence, similar to our results for VATS lobectomy in patients with small, early stage NSCLC.

The perceived complexity of segmentectomy relative to lobectomy and concerns regarding increased morbidity related to prolonged air leak and local recurrence rates have deterred most surgeons from using this approach for early stage lung cancer. Combining thoracoscopy and segmentectomy potentially increases these risks by increasing the complexity of the operation. Since 1973, when the first study was published suggesting that segmental resection was equivalent to lobectomy and therefore an adequate operation for stage I NSCLC, ${ }^{1}$ sublobar resection has been the topic of extensive debate. Some published studies have argued against segmentectomy as a procedure for early lung cancer. ${ }^{2,16,17}$ Ginsberg and colleagues ${ }^{2}$ published results showing the local recurrence rate to be 3 times higher after limited resec-
TABLE 5. Multivariate analysis of factors predicting recurrence Among patients with stage I non-small cell lung cancer

\begin{tabular}{lccc}
\hline \multicolumn{1}{c}{ Variable } & Hazard ratio & $\begin{array}{c}\mathbf{9 5} \% \text { Confidence } \\
\text { interval }\end{array}$ & $\begin{array}{c}\boldsymbol{P} \\
\text { value }\end{array}$ \\
\hline Type of resection & & & \\
$\quad$ Lobectomy & Reference & - & - \\
$\quad$ Segmentectomy & 0.85 & $0.21-3.51$ & .82 \\
Age & 0.96 & $0.91-1.01$ & .08 \\
Sex & & & \\
$\quad$ Female & Reference & - & - \\
$\quad$ Male & 1.06 & $0.42-2.71$ & .90 \\
Tumor size & 1.21 & $0.75-1.97$ & .43 \\
Histologic type & & & \\
Adenocarcinoma & Reference & - & - \\
Squamous cell carcinoma & 0.23 & $0.04-1.17$ & .08 \\
Other & 2.12 & $0.55-8.14$ & .28 \\
No. nodal stations sampled & 0.76 & $0.45-1.28$ & .30 \\
No. lymph nodes sampled & 1.07 & $0.95-1.20$ & .25 \\
T classification & & & \\
T1 & Reference & - & - \\
T2 & 4.49 & $1.36-14.90$ & .01 \\
\hline
\end{tabular}

tion than after lobectomy in patients with stage I NSCLC, with a resultant $30 \%$ increase in overall death rate. This study has been criticized for including about a third of patients with nonanatomic wedge resections in the limited resection group. Since then, numerous published studies have suggested that nonanatomic wedge resections result in higher rates of locoregional recurrence and worse cancer-related survival than anatomic resections, ${ }^{18,19}$ Thus combining patients undergoing wedge resection and with those undergoing segmentectomy potentially obscured any benefit limited to the patients undergoing segmentectomy.

In our study, patients undergoing VATS segmentectomy were discharged after stays similar in length to those of

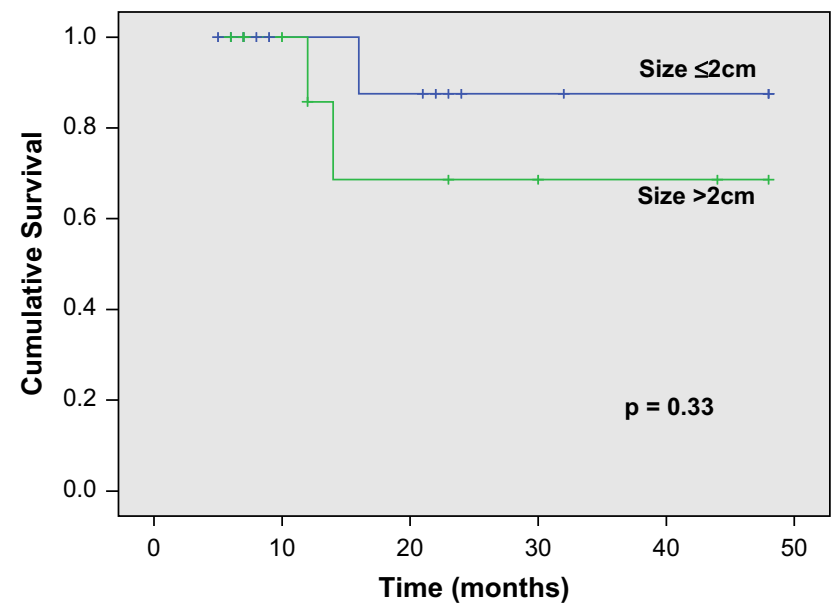

FIGURE 2. Disease-free survival in patients with pathologic stage I nonsmall cell cancer after video-assisted thoracoscopic surgery segmentectomy according to tumor size: $2 \mathrm{~cm}$ or smaller versus larger than $2 \mathrm{~cm}$. 
patients after VATS lobectomy, despite the fact that the patients in the VATS segmentectomy group had significantly worse pulmonary function before surgery. The median chest tube duration of 2 days and hospital stay of 4 days in our VATS segmentectomy group compares favorably with previously published results. ${ }^{3,7,14,20,21}$ Our results revealed a trend toward earlier chest tube removal after VATS segmentectomy but did not demonstrate any significant differences in chest tube duration and hospital stay between the groups. Furthermore, patients in both groups showed similar complication rates (VATS segmentectomy $25.8 \%$, VATS lobectomy $26.6 \%$ ). These rates are comparable to those in previously published reports, in which the average rates of complication are $17.6 \%$ to $31.3 \%$ after VATS segmentectomy, $32 \%$ to $39 \%$ after open segmentectomy, and $15.3 \%$ to $23.8 \%$ after VATS resection. ${ }^{8,14,20-22}$ There were 4 patients in our VATS segmentectomy group who underwent conversion to minithoracotomy, with only 1 of these conversions occurring as a result of bleeding. Two of the conversions occurred during the first 2 years of our 6-year experience, underscoring that VATS segmentectomy is a technically challenging procedure requiring a certain level of technical expertise in thoracoscopic procedures.

In the last decade, a growing number of published studies have demonstrated that segmental resections achieve comparable oncologic and survival outcomes to lobectomy. ${ }^{3-7,21}$ Schuchert and colleagues ${ }^{3}$ published results of 182 segmentectomies (done with thoracotomy and VATS) and 246 lobectomies in patients with NSCLC stage IA and IB. Similar overall recurrence rates were observed after segmentectomy $(17.6 \%)$ and lobectomy $(16.7 \%)$. There was also no difference in overall survival between the two groups. ${ }^{3}$

Our recurrence rates and mortalities are consistent with previously published data. Our overall recurrence rate in the VATS segmentectomy group was $17.2 \%$, versus $20.4 \%$ in the VATS lobectomy group. Furthermore, the locoregional recurrence rates were very low in our patients, $3.5 \%$ and $3.6 \%$ for VATS segmentectomy and VATS lobectomy respectively. The results of this study and the described recent studies suggest that at least for current tumors, which may be smaller and of a different histologic type than in earlier eras, thoracoscopic segmentectomy may be an acceptable operation from an oncologic standpoint.

Recently, more evidence has been published to suggest that tumor size plays an important role in recurrence among patients undergoing segmentectomy for early stage lung cancer. ${ }^{3-6,16}$ Okumura and colleagues ${ }^{4}$ reported a significant difference in survival among patients with T1N0M0 disease when tumor size exceeded $2 \mathrm{~cm}$, with 5-year survivals of $58 \%$ after segmentectomy and $78 \%$ after lobectomy. ${ }^{4}$ Schuchert and colleagues, ${ }^{3}$ however, have suggested that the ratio of margin to tumor is more important as a risk factor for recurrence than the size of the tumor alone. In their study, ratios of margin to tumor less than 1 are associated with a higher rate of recurrence. It becomes more difficult to maintain an adequate tumor-free margin as tumors get larger, which may help to explain the observation of a higher rate of recurrence among patients with larger lesions. We were unable to demonstrate a significant difference in recurrence rate for larger tumors. When we compared disease-free survival after VATS segmentectomy between patients with tumors $2 \mathrm{~cm}$ or smaller and those with tumors larger than $2 \mathrm{~cm}$, however, there was a trend toward decreased recurrence among patients with smaller lesions (Figure 2).

We have also demonstrated that an adequate lymph node dissection, which is important for proper staging and possibly survival, can be performed during VATS segmentectomy. The importance of an adequate assessment of pulmonary lymph nodes in patients with NSCLC is also supported by previously published studies. ${ }^{17,23-26}$

Moreover, as many as $11.5 \%$ of patients undergoing surgery for lung cancer have additional primary cancers develop within their lifetimes and thus require additional resection. ${ }^{17}$ Limited pulmonary resection allows future resections through the preservation of lung volume.

Because this was a retrospective study, the selection of patients was not random, which may have introduced a bias in the selection of a patient for a given procedure. Because VATS segmentectomy is not frequently done, the study had a low power to detect all clinically significant differences in recurrence between the two groups. The sparse data should be further assessed in future studies. The study being conducted by the Cancer and Leukemia Group B (CALGB 140503) should further elucidate the role of segmentectomy for patients with NSCLC $2 \mathrm{~cm}$ or smaller. It randomly assigns patients to undergo segmentectomy or wedge resection and lobectomy, with the access technique selected by the surgeon (VATS or thoracotomy).

In conclusion, this study suggests that thoracoscopic segmentectomy is a safe option for experienced thoracoscopic surgeons who are treating patients with small stage I lung cancers. In this retrospective study, there was no significant difference detected in oncologic outcome between VATS segmentectomy and VATS lobectomy groups. Furthermore, the lymph node dissection could be performed as effectively during VATS segmentectomy as during a VATS lobectomy. With experience, minimally invasive strategies can be applied to ever more challenging operations, such as pulmonary segmentectomy.

\section{References}

1. Jensik RJ, Faber LP, Milloy FJ, Monson DO. Segmental resection for lung cancer. A fifteen-year experience. J Thorac Cardiovasc Surg. 1973;66:563-72.

2. Ginsberg RJ, Rubinstein LV. Randomized trial of lobectomy versus limited resection for T1 N0 non-small cell lung cancer. Lung Cancer Study Group. Ann Thorac Surg. 1995;60:615-23. 
3. Schuchert MJ, Pettiford BL, Keeley S, D'Amato TA, Kilic A, Close J, et al. Anatomic segmentectomy in the treatment of stage I non-small cell lung cancer. Ann Thorac Surg. 2007;84:926-33.

4. Okumura M, Goto M, Ideguchi K, Tamura M, Sasaki H, Tanaka H, et al. Factors associated with outcome of segmentectomy for non-small cell lung cancer: longterm follow-up study at a single institution in Japan. Lung Cancer. 2007;58:231-7.

5. El-Sherif A, Gooding WE, Santos R, Pettiford B, Ferson PF, Fernando HC, et al. Outcomes of sublobar resection versus lobectomy for stage I non-small cell lung cancer: a 13-year analysis. Ann Thorac Surg. 2006;82:408-16.

6. Okada M, Yoshikawa K, Hatta T, Tsubota N. Is segmentectomy with lymph node assessment an alternative to lobectomy for non-small cell lung cancer of $2 \mathrm{~cm}$ or smaller? Ann Thorac Surg. 2001;71:956-61.

7. Martin-Ucar AE, Nakas A, Pilling JE, West KJ, Waller DA. A case-matched study of anatomical segmentectomy versus lobectomy for stage I lung cancer in highrisk patients. Eur J Cardiothorac Surg. 2005;27:675-9.

8. Onaitis MW, Petersen RP, Balderson SS, Toloza E, Burfeind WR, Harpole DH Jr, et al. Thoracoscopic lobectomy is a safe and versatile procedure: experience with 500 consecutive patients. Ann Surg. 2006;244:420-5.

9. Nagahiro I, Andou A, Aoe M, Sano Y, Date H, Shimizu N. Pulmonary function, postoperative pain, and serum cytokine level after lobectomy: a comparison of VATS and conventional procedure. Ann Thorac Surg. 2001;72:362-5.

10. Nicastri DG, Wisnivesky JP, Litle VR, Yun J, Chin C, Dembitzer FR, et al. Thoracoscopic lobectomy: report on safety, discharge independence, pain, and chemotherapy tolerance. J Thorac Cardiovasc Surg. 2008;135:642-7.

11. Ohtsuka T, Nomori H, Horio H, Naruke T, Suemasu K. Is major pulmonary resection by video-assisted thoracic surgery an adequate procedure in clinical stage I lung cancer? Chest. 2004;125:1742-6.

12. Walker WS, Codispoti M, Soon SY, Stamenkovic S, Carnochan F, Pugh G. Longterm outcomes following VATS lobectomy for non-small cell bronchogenic carcinoma. Eur J Cardiothorac Surg. 2003;23:397-402.

13. Petersen RP, Pham D, Burfeind WR, Hanish SI, Toloza EM, Harpole DH Jr, et al. Thoracoscopic lobectomy facilitates the delivery of chemotherapy after resection for lung cancer. Ann Thorac Surg. 2007;83:1245-50.

14. Shiraishi T, Shirakusa T, Iwasaki A, Hiratsuka M, Yamamoto S, Kawahara K. Video-assisted thoracoscopic surgery (VATS) segmentectomy for small peripheral lung cancer tumors: intermediate results. Surg Endosc. 2004;18:1657-62.
15. Shiraishi T, Shirakusa T, Miyoshi T, Hiratsuka M, Yamamoto S, Iwasaki A. A completely thoracoscopic lobectomy/segmentectomy for primary lung cancertechnique, feasibility, and advantages. Thorac Cardiovasc Surg. 2006;54: 202-7.

16. Warren WH, Faber LP. Segmentectomy versus lobectomy in patients with stage I pulmonary carcinoma. Five-year survival and patterns of intrathoracic recurrence. J Thorac Cardiovasc Surg. 1994;107:108-94.

17. Martini N, Bains MS, Burt ME, Zakowski MF, McCormack P, Rusch VW, et al Incidence of local recurrence and second primary tumors in resected stage I lung cancer. J Thorac Cardiovasc Surg. 1995;109:120-9.

18. Landreneau RJ, Sugarbaker DJ, Mack MJ, Hazelrigg SR, Luketich JD, Fetterman L, et al. Wedge resection versus lobectomy for stage I (T1 N0 M0) non-small-cell lung cancer. J Thorac Cardiovasc Surg. 1997;113:691-700.

19. Sienel W, Dango S, Kirschbaum A, Cucuruz B, Horth W, Stremmel C, et al. Sublobar resections in stage IA non-small cell lung cancer: segmentectomies result in significantly better cancer-related survival than wedge resections. Eur J Cardiothorac Surg. 2008;33:728-34.

20. Atkins BZ, Harpole DH Jr, Mangum JH, Toloza EM, D'Amico TA, Burfeind WR Jr. Pulmonary segmentectomy by thoracotomy or thoracoscopy: reduced hospital length of stay with a minimally-invasive approach. Ann Thorac Surg. 2007;84:1107-13.

21. Jones DR, Stiles BM, Denlinger CE, Antippa P, Daniel TM. Pulmonary segmentectomy: results and complications. Ann Thorac Surg. 2003;76:343-9.

22. McKenna RJ Jr, Houck W, Fuller CB. Video-assisted thoracic surgery lobectomy: experience with 1,100 cases. Ann Thorac Surg. 2006;81:421-6.

23. Ludwig MS, Goodman M, Miller DL, Johnstone PA. Postoperative survival and the number of lymph nodes sampled during resection of node-negative non-small cell lung cancer. Chest. 2005;128:1545-50.

24. Ou SH, Zell JA. Prognostic significance of the number of lymph nodes removed at lobectomy in stage IA non-small cell lung cancer. J Thorac Oncol. 2008;3:880-6.

25. Doddoli C, Aragon A, Barlesi F, Chetaille B, Robitail S, Giudicelli R, et al. Does the extent of lymph node dissection influence outcome in patients with stage I nonsmall-cell lung cancer? Eur J Cardiothorac Surg. 2005;27:680-5.

26. Miller DL, Rowland CM, Deschamps C, Allen MS, Trastek VF, Pairolero PC Surgical treatment of non-small cell lung cancer $1 \mathrm{~cm}$ or less in diameter. Ann Thorac Surg. 2002;73:1545-51. 